\title{
Ensino e prática de enfermagem em emergência: apoio tecnológico
}

\section{Teaching and practice of nursing in emergency: technological support}

\author{
${ }^{1}$ Douglas Markonne de Souza Santos dmarkonneadm@gmail.com \\ ${ }^{1}$ Ilda Cecilia Moreira da Silva \\ ${ }^{1}$ Lucrécia Helena Loureiro \\ ${ }^{2}$ Annibal José Roris Rodriguez Scavarda
}

\section{RESUMO}

Objetivou-se realizar uma revisão integrativa da literatura sobre os tipos de tecnologias que enfermeiros têm desenvolvido ou que os mesmos poderiam utilizar para a assistência de enfermagem em emergências. Realizou-se de abril a junho de 2017 busca com os descritores: "tecnologia em saúde”, "assistência de enfermagem”; "emergência”, "educação em enfermagem nas bases: SciELO, PubMed, BVS, BDENF, BDTD/UFMG, PERIODICOS CAPES/MEC, em periódicos brasileiros. Encontrou-se 7938 referências, publicadas no idioma português e 687 em inglês, das quais trinta atenderem aos critérios de seleção do estudo, $47 \%$ português e $53 \%$ em inglês. Segundo a classificação do Qualis, $30 \% \mathrm{~A}^{1} ; 27 \% \mathrm{~B}^{1} ; 17 \% \mathrm{~B}^{2}$; $13 \% \mathrm{~A}^{2} ; 10 \% \mathrm{C} ; 3 \% \mathrm{~B}^{4}$, comprovando o padrão de qualidade excelente nos estudos desenvolvidos pela Enfermagem acerca da temática. Identificou-se três tipos de tecnologias para o cuidado, classificadas como leve, leve-dura e dura. As tecnologias leve e leve-dura as mais utilizadas, por serem práticas facilmente desenvolvidas e aplicáveis no gerenciamento do cuidado. Conclui-se que a enfermagem, por intermédio de suas inovações tecnológicas é capaz de avançar na aplicação dessas tecnologias, articuladas especialmente às ações de ensinar, gerenciar e cuidar em saúde.

Palavras-chave: Tecnologias em saúde. Emergência. Assistência de enfermagem. Educação em enfermagem.

\begin{abstract}
The objective was to carry out an integrative review of the literature on the types of technologies that nurses have developed or that they could use for nursing assistance in emergencies. From April to June of 2017, the search was carried out with the following descriptors: "technology in health", "nursing care"; "Emergency", "nursing education at the bases: SciELO, PubMed, BVS, BDENF, BDTD / UFMG, PERIODICOS CAPES / MEC, in Brazilian periodicals. There were 7938 references, published in the portuguese language and 687 in English, of which 30 met the study selection criteria, 47\% Portuguese and 53\% in English. According to the Qualis classification, 30\% A1; 27\% B1; 17\% B2; 13\% A2, 10\% C; 3\% B4, confirming the excellent quality standard in the studies developed by Nursing on the subject. Three types of care technologies were identified, classified as light, light-hard and hard. The lightweight and hard technologies are the most commonly used because they are easily developed and applicable practices in the management of care. It is concluded that nursing, through its technological innovations is able to advance in the application of these technologies, especially articulated to the actions of teaching, managing and health care.
\end{abstract}

Keywords: Health technologies. Emergency. Nursing care. Education in nursing.

1 Centro Universitário de Volta Redonda - UniFOA

2 UNIRIO 


\section{INTRODUÇÃO}

O extraordinário progresso da ciência e principalmente da informática, que nas últimas décadas afetou todos os setores da economia formal e informal, não é diferente na enfermagem que acompanhou esse progresso. Tratar a complexidade da inovação tecnológica não é uma tarefa fácil, entretanto, pesquisas vêm demonstrando que a cada dia as instituições de saúde utilizam e beneficiam-se com a variedade de aplicativos pensados para esses espaços de cuidar.

De acordo com Rocha et al. (2008) entre os séculos XVIII e XIX, iniciou a primeira revolução técnico-cientifica das grandes transformações com a substituição da força física produtiva do homem às energias das máquinas a vapor e a eletricidade. Na sequência, a revolução industrial e a segunda guerra mundial viabilizaram o entrecruzamento entre ciência e tecnologias, passando a servir de equipamentos mais modernos e aprimorados, aumentando a produção dos produtos e serviços.

Nietsche et al. (2014) chama a atenção que na Europa nesse mesmo período histórico, ocorria um movimento social de atividades tecnológicas com objetivo de estruturar os diversos ramos da ciência que passavam a incluir as teorias, os métodos e os processos científicos para soluções de problemas técnicos. Por outro lado, na Inglaterra a enfermagem profissional começava a despertar com Florence Nightingale, que influenciada pelas concepções do movimento tecnológico iniciou observações sistêmicas e registrava sua prática de cuidado com doentes. Ela criou quatro conceitos fundamentais balizadores: ser humano, meio ambiente, saúde e enfermagem.

Por seu intermédio, a enfermagem começou a globalizar-se cada vez mais, viabilizando a interação de enfermeiros em diferentes estados e países. No campo da saúde, as primeiras contribuições de produção tecnológica foram à introdução de instrumentos para atos cirúrgicos e equipamentos diagnósticos de finalidade terapêutica (Rocha et al.,2008).

Mundialmente, em destaque no Brasil, as descobertas de Florence Nightingale contribuíram para o alinhamento da construção de modelos conceituais para a investigação sistematizada de conhecimentos específicos. Dessa maneira, a expressão tecnologia ficou evidente no processo e no produto do pensar/fazer o cuidado, com a instituição da fundamentação cientifica (Rocha et al.,2008).

De acordo com Nietsche et al. (2014), existe o entrelaçamento entre o cuidado de enfermagem e as dimensões tecnológicas, “(...) o cuidado e a tecnologia estão também interligados, uma vez que a enfermagem está comprometida com princípios, leis e teorias, e a tecnologia consiste na expressão desse conhecimento cientifico e em sua própria transformação”. O entender da autora nos permite refletir acerca do cuidado na ótica tecnológica, e nos leva a repensar a inerente capacidade de produção cientifica e transformações sociais e de saúde que os enfermeiros são capazes. Dessa forma, alcançamos o entendimento de que o cuidado faz parte da materialização tecnológica da enfermagem, pois possibilita o desenvolvimento de projetos de saúde para pessoas e a coletividade.

Encontra-se na comunidade científica alguns autores da enfermagem que se dedicam a estudar sobre a temática “tecnologia em saúde”, entre eles, (Rossi \& Lima, 2005; Gonçalves \& Schier, 2005; Nietsche et al., 2014 e Loureiro et al.,2016), eles afirmam que as tecnologias em saúde são estratégias de inovação/aperfeiçoamento na prática de enfermagem nos diferentes campos de atuação, inclusive nas consultas de enfermagem.

No campo da saúde, particularmente na enfermagem, Rocha et al.(2008) entende que o cuidado se fundamenta no conhecimento "cientifico, técnico, pessoal, cultural, social, econômico, político e psicoespiritual”. Dessa maneira, a enfermagem desenvolve atitudes e comportamentos com o intuito de alcançar a "promoção, manutenção e ou recuperação da saúde, dignidade e totalidade humana”. 
Sabe-se que a enfermagem moderna se fundamenta na ciência do cuidado, desenvolve práticas objetivas de desenvolvimento técnico e procedimental e se baseia na criatividade e intuição Rocha et al. ( 2008). Os estudiosos declaram que as tecnologias nos processos de trabalho em saúde são evidenciadas pelo saber tecnológico que a enfermagem possui e realiza na prática, fundamentada no conhecimento específico desses profissionais. Neste caminho, estes autores afirmam que existem desafios para representar as tecnologias cuidativas-educacionais, em produzi-las, testá-las e avaliá-las. Corroborando com esta assertiva, Merhy, (2002 ) aponta para uma das possibilidades de simbolização das tecnologias em saúde, ao afirmar que tecnologias são incorporadas no processo de trabalho dos profissionais de saúde.

Merhy, se utiliza dessas alegações e da natureza instigante e estimulante da temática em destaque, assim, surgiu o interesse em encontrar possíveis soluções de tecnologias utilizadas pelo enfermeiro na pratica do cuidar no setor de emergência.

Neste sentido, Rocha et al. (2008), classificam a tecnologia em: tecnologia de produto e tecnologia de processo. Em contra partida Merhy 2002; Rocha et al.(2008), classifica em três tipificações; leve (quando se fala em relações, acolhimento e gestão), leve-dura (ao se referir a saberes estruturado, como o processo de enfermagem) e a dura (envolvendo equipamentos, máquinas e normas).

A tecnologia gera conhecimentos socializados, governa processos e produtos, transforma-os numa aplicação empírica de modo a torná-la científica, Nietsche et al. (2014). Um estudo sobre tecnologias em saúde poderia contemplar inúmeras possibilidades de busca, tais como, aspectos históricos da tecnologia, produção e aplicação de tecnologias no sistema de saúde brasileiro ou tecnologias socioeducativas na área hospitalar. Entretanto, optou-se por enfatizar a busca de tecnologias utilizadas pela enfermagem no setor de emergência.

De um modo geral, no cotidiano alguns modelos de atenção à saúde apresentam característica mecanicista, privilegiando a fragmentação do saber por meio da centralidade no atendimento médico principalmente nas emergências hospitalares.

Esse modelo tradicional biomédico adotado nas principais portas de entrada das emergências do SUS apresentam resultados insuficientes e pouco satisfatórios de expectativas de saúde pela população brasileira. Nessa perspectiva, questiona se a existência de soluções tecnológicas para assistência de enfermagem nas emergências.

No intuito de buscar evidências de inovações tecnológicas apropriadas “do modo de pensar e fazer enfermagem” nos diversos campos de atuação, este estudo pretende alcançar o serviço de emergência.

Nesse aspecto, o estudo teve por objetivo identificar as tecnologias em saúde direcionadas à enfermagem nos serviços de emergência. Espera-se que possa colaborar para uma reflexão crítica da prática assistencial da enfermagem em emergência, objetivando aprimorar a atuação dos enfermeiros. Assim, uma assistência que agrega tais informações possibilitará ao enfermeiro um planejamento mais adequado de suas ações, além de contribuir para ampliação da autonomia, inovação na prática profissional e melhoria na qualidade da assistência prestada.

\section{METODOLOGIA}

\section{1 Desenho, local do estudo e período}

Trata-se de um estudo de abordagem qualitativa sob a forma de revisão integrativa, um método de pesquisa que possibilita a síntese de estudos publicados e geram deduções gerais a respeito de uma determinada área de pesquisa. A revisão integrativa da literatura (RIL) proporciona conclusões sobre o problema de pesquisa, identificando as lacunas do conhecimento em relação ao fenômeno em estudo. De acordo com Whittemore et al. ( 2005) , a RIL é um método amplo que permite a inclusão de literatura teórica e empírica, bem como estudos de 
abordagem qualitativa. Essa técnica permite atualizar as discussões relacionadas a um tema especifico, a partir da síntese de estudos publicados. Para construção desta revisão, foram percorridos as seis etapas propostas por Pompeo et al. (2009), a identificação do tema, amostragem, identificação, pré - seleção/ seleção dos estudos , categorização, análise, interpretação e síntese do conhecimento evidenciado nos artigos. Dessa forma, inicialmente formulou-se a seguinte questão norteadora: Quais os tipos de tecnologias em saúde são utilizados pela enfermagem nos serviços de emergência?

O levantamento bibliográfico foi realizado por meio de consulta nas bases de dados SciELO - Scientific Electronic Library, PubMed - US National Library of Medicine National Institutes of Health, BVS - Biblioteca Virtual em Saúde, BDENF - Banco de Dados em Enfermagem, BDTD/UFMG - Biblioteca Digital de Teses e Dissertação da Universidade Federal de Minas Gerais, Portal de Periódicos CAPES/MEC - Ministério da Educação. Escolhemos esta estratégia de pesquisa, devido a triagem detalhada e objetividade de apuração das informações manifestadas. Tal busca ocorreu nos meses de janeiro a junho de 2017.

\section{2 Amostra e critérios de inclusão e exclusão}

Nas bases de dados, formam utilizados os seguintes descritores: tecnologias em saúde, emergência, assistência de enfermagem, educação em enfermagem; de acordo com a terminologia DeCS - Descritores em Ciências da Saúde. A equação de busca foi "tecnologia em saúde” AND "emergência” AND “ assistência de enfermagem” AND “ educação em enfermagem”

Os critérios de inclusão delimitados para pré-seleção dos estudos foram: ser artigo produzido por enfermeiros; contemplar o objetivo proposto; ter sido publicado no período de 2012 a 2016; estar no idioma português ou inglês e estar disponível eletronicamente na integra. Foram excluídos os editoriais, cartas ao editor, trabalhos publicados em anais de eventos, textos incompletos, em outros idiomas e artigos repetidos.

Para descrição das buscas e seleção dos estudos utilizou-se a Preferred Reporting Itens for Systematic Review and Meta-Analyses ( PRISMA), conforme fluxograma a seguir ( Figura 1 e 2 ) 
Figura 1 - Processo de seleção dos estudos no idioma português.

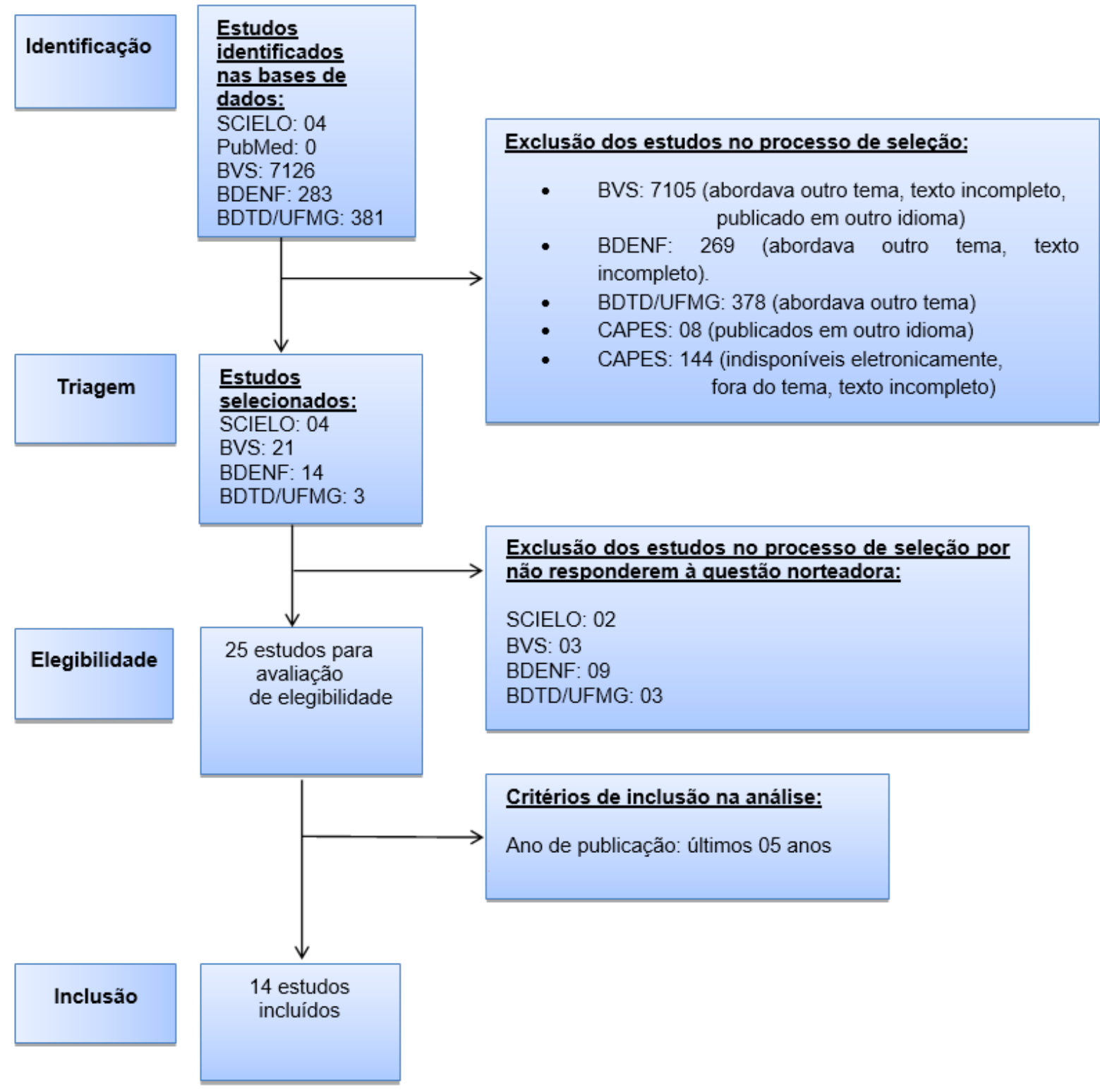

Fonte: Dos autores, 2017. 
Figura 02 - Processo de seleção dos estudos publicados no idioma inglês.

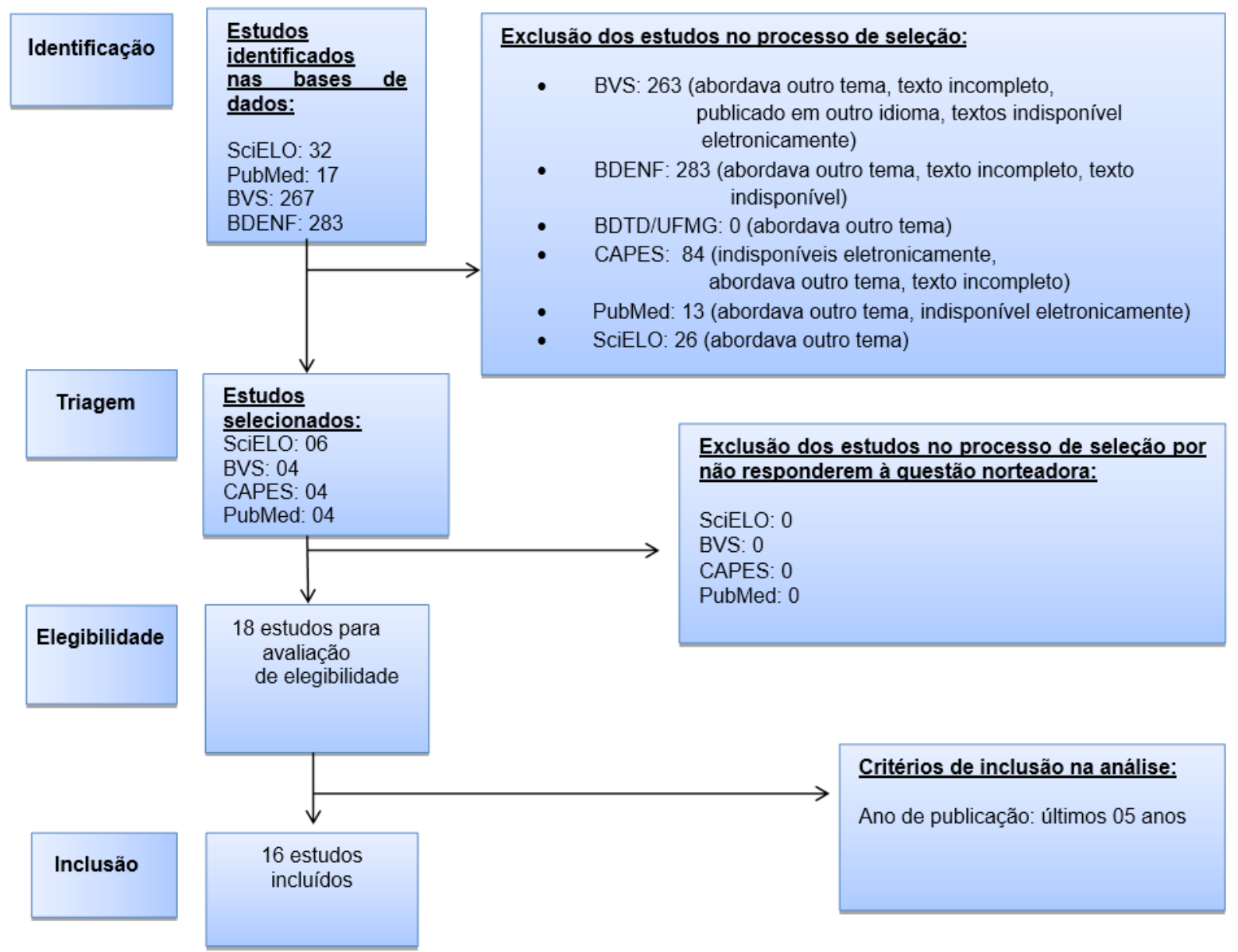

Fonte: Dos autores, 2017.

\section{3 Fase 4: Categoria dos estudos selecionados: matriz de síntese}

O processo de seleção dos estudos foi executado por meio da leitura minuciosa de títulos e resumos, de modo que foram para seleção final os estudos que atendiam aos critérios de inclusão supracitados. Ao final foram selecionados doze artigos da base da BVS; oito da SCIELO; quatro da BDENF; quatro do PUBMED; dois da CAPES. Dessa forma, a revisão foi composta por 30 artigos.

\section{RESULTADOS E DISCUSSÃO}

Os resultados foram apresentados sob a forma de quadro e gráficos; analisados de acordo com o método de análise de conteúdo de Bardin et al. (1977), que permitiu o agrupamento do conteúdo estudado em categorias temáticas e a análise foi desenvolvida em três etapas:

a. Etapa 1 ( pré - exploração do material) foram realizadas leituras flutuantes dos artigos selecionados com o objetivo de conhecer o contexto e abstrair impressões relevantes.

b. Etapa 2 ( seleção das unidades de análise) nesta fase foram destacadas sentenças e frases que se apresentavam com maior frequência no intuito de construir unidades temáticas. 
c. Etapa 3 (categorização dos estudos) realizou-se leitura profunda do material distribuído nas categorias, foram expresso os significados e as interpretações abstraídas no intuito de construir novos conhecimentos. (Campos et al., 2004)

Na caracterização dos artigos, observou-se que doze (40\%) da BVS; oito (27\%) SciELO; quatro (13\%) Pubmed; quadro (13\%) BEDENF e dois (7\%) Periódicos da CAPES. A abordagem que mais prevaleceu foi a qualitativa, com quinze (50\%) publicações; seguindo de dez (33\%) estudos quantitativos; e cinco (17\%) método misto.

Após a leitura exaustiva dos artigos na íntegra criamos o quadro de análise e dividimos a biblioteca individual em gavetas e pastas; as gavetas são os blocos contendo o tipo de texto, idioma, periódicos, qualis dos periódicos e a tecnologia em saúde utilizada. Pastas estão contidas dentro das gavetas e correspondem aos estudos encontrados na pesquisa e analisados para discussão dos resultados. A estrutura tecnológica se dividiu em leve, leve-dura e dura, que propiciaram a discussão acerca dos tipos de tecnologias utilizadas no cotidiano da enfermagem dentro do serviço de emergência.

No que tange ao ano de publicação, constatou-se que, no último quinquênio, destacou-se o ano 2012 com nove artigos (30\%), seguido de 2016 com oito artigos (23\%), 2013 com seis artigos (20\%), 2014 com quatro artigos (14\%) e 2015 com três artigos (14\%). Existe uma relação intima entre a quantidade numerosa identificada de artigos produzidos no primeiro ano da análise e dispositivos legais que tratam sobre o tema; elaborados pelo Conselho Federal de Enfermagem e pelo Ministério da Saúde.

O Conselho Federal de Enfermagem - COFEN normatizou a resolução nº 423/2012 que trata, no âmbito da equipe de Enfermagem, a classificação de risco e priorização da assistência em urgências e emergências como privativas do enfermeiro. Outrossim, o profissional precisa ter conhecimento, competência e habilidade técnico-cientifica para executar o procedimento.

Nesse mesmo ano, o Ministério da Saúde implementou a portaria nº 1.663, em 6 de agosto, que dispõe sobre o programa SOS Emergência, na esfera da rede de atenção às urgências e emergências. O projeto ministerial objetiva a melhoria da gestão e qualidade assistencial, adequação física e tecnológica dos serviços hospitalares de emergência; por meio da classificação de risco, gestão de leitos, protocolos clínico-assistenciais e administrativos, regulação e articulação com o sistema de saúde (Brasil, 2012).

Segundo a classificação do Qualis, nove (30\%) A ${ }^{1}$; oito ( $\left.27 \%\right) B^{1}$; cinco (17\%) B ; quatro (13\%) A ; três ( 10\%) C; um ( 3\%) B ${ }^{4}$; comprovando o padrão de qualidade excelente nos estudos desenvolvidos pela Enfermagem acerca da temática. Quanto aos periódicos com maior número de artigos publicados relacionados a temática, destacaram-se: a revista Latino Americana de Enfermagem ( RLAE) com quatro artigos (14,1\%), seguidos da revista Investigação, Educação e Enfermagem com três artigos ( Quadro 1 ).

Figura 3 - Recorte temporal das publicações referente ao quinquênio de 2012 a 2016 (n = 30).

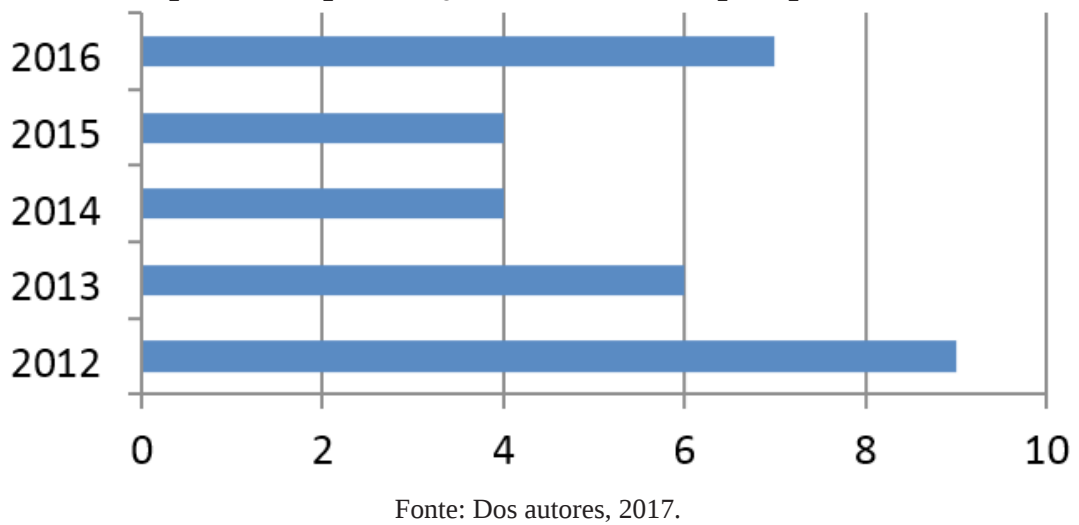


Quadro 01- Síntese dos estudos incluídos na revisão integrativa (n= 30).

\begin{tabular}{|c|c|c|c|c|}
\hline$N^{o}$ & Títulos & Autor (es) & $\begin{array}{l}\text { Ano/ } \\
\text { base de } \\
\text { dados }\end{array}$ & $\begin{array}{l}\text { Delineamento do } \\
\text { estudo }\end{array}$ \\
\hline 01 & $\begin{array}{l}\text { Análise do perfil do paciente como } \\
\text { vítima de múltiplos traumas }\end{array}$ & $\begin{array}{c}\text { Bertoncello; } \\
\text { Cavalcanti; Ilha }\end{array}$ & $\begin{array}{l}\text { 2012/ } \\
\text { BVS }\end{array}$ & $\begin{array}{l}\text { Quantitativo, exploratório, } \\
\text { descritivo, transversal }\end{array}$ \\
\hline 02 & $\begin{array}{l}\text { Um olhar a qualidade no processo } \\
\text { de atendimento em um serviço } \\
\text { de urgência público }\end{array}$ & Silva; Matsuda & $\begin{array}{c}\text { 2012/ } \\
\text { BDENF }\end{array}$ & Qualitativa \\
\hline 03 & $\begin{array}{l}\text { Equipe de enfermagem e detecção } \\
\text { de indicadores de agravamento em } \\
\text { pacientes de pronto-socorro }\end{array}$ & Jorge et al. & $\begin{array}{c}\text { 2012/ } \\
\text { SciELO }\end{array}$ & $\begin{array}{l}\text { Qualitativa, exploratório, } \\
\text { descritivo. }\end{array}$ \\
\hline 04 & $\begin{array}{l}\text { Atendimento à crise psíquica } \\
\text { no pronto-socorro: visão de } \\
\text { profissionais de enfermagem }\end{array}$ & Borges et al. & $\begin{array}{c}\text { 2012/ } \\
\text { SciELO }\end{array}$ & Qualitativa \\
\hline 05 & $\begin{array}{l}\text { Sistematização da assistência de } \\
\text { enfermagem em serviços de urgência e } \\
\text { emergência: viabilidade de implantação }\end{array}$ & Maria; Quadros; Grassi & $\begin{array}{c}\text { 2012/ } \\
\text { SciELO }\end{array}$ & $\begin{array}{l}\text { Qualitativo, descritivo, } \\
\text { estudo de campo }\end{array}$ \\
\hline 06 & $\begin{array}{l}\text { Implementação da classificação de } \\
\text { risco em unidade de emergência } \\
\text { deum hospital público do Rio de } \\
\text { Janeiro - um relato de experiência }\end{array}$ & Junior et al. & $\begin{array}{c}\text { 2012/ } \\
\text { BDENF }\end{array}$ & $\begin{array}{l}\text { Quantitativo, relato de } \\
\text { experiência, descritivo }\end{array}$ \\
\hline 07 & $\begin{array}{l}\text { Predictivevalidityofthe Manchester } \\
\text { Triage System: evaluationofoutcomes } \\
\text { ofpatientsadmittedtoan } \\
\text { emergencydepartment }\end{array}$ & $\begin{array}{l}\text { Domingos; Salgado; } \\
\text { Chianca }\end{array}$ & $\begin{array}{l}\text { 2012/ } \\
\text { BVS }\end{array}$ & Quantitativo, coorte prospectivo \\
\hline 08 & $\begin{array}{l}\text { Occupational stress of nurse in } \\
\text { emergenycare: an integrative } \\
\text { review of the literature }\end{array}$ & Bezerra; Silva; Ramos & $\begin{array}{l}\text { 2012/ } \\
\text { BVS }\end{array}$ & $\begin{array}{c}\text { Revisão integrativa da } \\
\text { literatura }\end{array}$ \\
\hline 09 & $\begin{array}{l}\text { Conceptionsof nurses on the management } \\
\text { of care in anemergencyservice: } \\
\text { descriptive-exploratory study }\end{array}$ & Santos et al. & $\begin{array}{l}\text { 2012/ } \\
\text { BVS }\end{array}$ & $\begin{array}{l}\text { Qualitativo, exploratório, } \\
\text { descritivo }\end{array}$ \\
\hline 10 & $\begin{array}{l}\text { Nursingwork in care practiveatemergency } \\
\text { careunits in Porto Alegre-RS }\end{array}$ & Gehlen; Lima & $\begin{array}{l}\text { 2013/ } \\
\text { BVS }\end{array}$ & Quantitativo, Qualitativo \\
\hline 11 & $\begin{array}{l}\text { Nurse'scareand managementactions } \\
\text { in emergency trauma cases }\end{array}$ & $\begin{array}{l}\text { Azevedo; Scarparo; } \\
\text { Chaves }\end{array}$ & $\begin{array}{l}\text { 2013/ } \\
\text { BVS }\end{array}$ & $\begin{array}{l}\text { Qualitativo, exploratório, } \\
\text { descritivo, casos múltiplos }\end{array}$ \\
\hline 12 & $\begin{array}{l}\text { The quality of nursing care at na } \\
\text { emergencyunit: the patient’sperception }\end{array}$ & Morais; Melleiro & $\begin{array}{c}\text { 2013/ } \\
\text { CAPES }\end{array}$ & $\begin{array}{l}\text { Quantitativo, exploratório, } \\
\text { descritivo. }\end{array}$ \\
\hline 13 & $\begin{array}{l}\text { Publichealthemergency: } \\
\text { socialrepresentations among } \\
\text { managers of a university hospital }\end{array}$ & Souza; Dall’ Agnol & $\begin{array}{c}\text { 2013/ } \\
\text { PubMed }\end{array}$ & Qualitativo, estudo de caso \\
\hline 14 & $\begin{array}{l}\text { Avaliação da qualidade das } \\
\text { anotações de enfermagem no pronto } \\
\text { atendimento de um hospital escola }\end{array}$ & Seignemartinet al. & $\begin{array}{l}\text { 2013/ } \\
\text { BVS }\end{array}$ & $\begin{array}{l}\text { Quantitativo, retrospectivo, } \\
\text { transversal }\end{array}$ \\
\hline 15 & $\begin{array}{l}\text { Atuação de enfermagem em serviços } \\
\text { de emergência: revisão sistemática. }\end{array}$ & Sobral et al. & $\begin{array}{l}\text { 2013/ } \\
\text { BVS }\end{array}$ & $\begin{array}{l}\text { Revisão sistemática } \\
\text { Sem metanalise }\end{array}$ \\
\hline
\end{tabular}


16

Dor torácica: atuação do enfermeiro em um pronto atendimento de um hospital escola.

17

Knowledge of cardiopulmonary

resuscitationamong pediatricemergency staff

Cuidados intensivos em um

18 serviço hospitalar de emergência:

desafiospara os enfermeiros

19

Assessmentandrisk classificationprotocol

for patients in emergency units

Caracterização das vítimas de

20 traumas por acidente com motocicleta

internada sem hospital público

21

Care management in nursing

withinemergencycareunits

Family'spresence in the

22 pediatricemergencyroom: opinionofhealth's professionals

Symptomsofdepressionand

23 interveningfactorsamong nurses

ofemergency hospital services

Percepção de enfermeiras (o)s sobre

24 acolhimento com classificação de risco no serviço de pronto atendimento.

25

O processo de trabalho do enfermeiro no acolhimento com classificação de risco

Manchester Triage System: main

26 flowcharts, discriminators andoutcomesof a pediatric emergency care

27

Perceptionofemergency nurses in using

a chestplain assessmentprotocol

28

Triage in anadult emergencyservice:

patient satisfaction

29 Elder-friendlyemergencyservices

inBrazil: necessaryconditions for care

30
2014/
Caveiãoet al. $\quad$ BVS

Bertolo et al.

2014/

BVS

Zandomenighi et al.

2014/

BDENF

Silva et al.

2014/

PubMed

Soares et al.

2015/

BVS

Oliveira et al.

Mekitarian; Angelo

Oliveira; Mazzaia;

Marcolan

2015/

SciELO

2016/

Prudêncio et al.

SciELO

Rates; Alves; Cavalcante

2016/

BDENF

Amthuer; Cunha

Vieira et al.

Silva et al.

Santos; Lima; Zucatti

Santos et al.
PubMed

2016/

PubMed

2016/

SciELO

2016/

SciELO

2016/ SciELO
Quantitativo, descritivo, exploratório

Quantitativo, transversal, descritivo

Quantitativo, qualitativo exploratório, transversal, descritivo

Quantitativo

Quantitativo, Descritivo

Qualitativo

Quantitativo, Qualitativo, transversal

Quantitativo, qualitativo, transversal

Qualitativo

Qualitativo, estudo de caso

Quantitativo, transversal

Qualitativo, Descritivo

Qualitativo, exploratório, transversal, descritivo

Quantitativo, qualitativo, descritivo

Qualitativo, estudo de caso 
Figura 4 - Quantitativo de artigos referentes as bases de dados pesquisadas $(n=30)$.



Fonte: Dos autores, 2017.

Os 30 trabalhos selecionados foram submetidos à análise de conteúdo e desvelaram-se em três categorias ou temas: Tecnologia leve em enfermagem: liderança situacional, trabalho em equipe e gestão de relacionamentos; Tecnologia leve-dura: classificação de risco, processo de enfermagem e educação permanente e Tecnologia dura em enfermagem: sistema de informação e protocolos assistenciais de apoio ao cuidado.

\section{1 Tecnologia leve em enfermagem: liderança situacional, trabalho em equipe e gestão de relaciona- mentos}

O desenvolvimento da maturidade adaptativa de tomar decisões rápidas e fundamentadas permita ao enfermeiro articular ações profissionais, estabelecer relações de cooperação, construir e manter vínculos amistosos e gerenciar conflitos. Essa capacidade de identificar múltiplos problemas e agir de forma variada é explicada pelos diferentes padrões de liderança que o ambiente hospitalar requer deste profissional.

As publicações se localizam, especialmente, no trabalho do gerente de enfermagem, que executa sua ocupação de forma coletiva e participativa; sua capacidade humana e profissional de trabalhar com pessoas é fundamental no serviço. Além disso, necessita desenvolver essas competências para promover a comunicação efetiva, o relacionamento interpessoal saudável, à mediação de interesses e articulação de decisões integrativas de cuidado.

Trazendo para o campo da emergência, segundo Sobral et al. (2013), a liderança situacional contribui para esse comportamento flexível e dinâmico; permiti instrumentalizar o enfermeiro a buscar modelos gerenciais distintos frente as necessidades de atuação em quanto gestor da equipe de enfermagem.

Nos textos analisados, articulação é utilizada como uma tecnologia leve pelos enfermeiros na integração das ações e potencialização do trabalho em equipe. O diálogo e a interação entre os profissionais são componentes do trabalho, aonde o agente elabora correlações e coloca em evidencia as conexões entre as melhores formas de tratar os relacionamentos (Santos et al. 2016).

Os discursos se orientam por um padrão ideal de comunicação, Morais et al.(2013), afirmam que, a satisfação é o produto da gestão dos relacionamentos e mediadora da qualidade da assistência, resulta da percepção da equipe de enfermagem e de pacientes atendidos. A comunicação e a informação clínica são elementos que influenciam no grau de satisfação do usuário.

Vale ainda destacar que, o cuidado gerencial realizado de forma segura permite estabelecer intervenções na gestão, e as decisões do cotidiano, podem ser interpretadas e consideradas como parâmetro de satisfação. 
Ademais, a segurança na prática gerencial promove sustentação para realização da assistência de maneira sistematizada.

\section{2 Tecnologia leve-dura: classificação de risco, processo de enfermagem e educação permanente}

Algumas publicações contribuem para o entendimento do atendimento de enfermagem, baseado no saber técnico e científico, realizado pelo enfermeiro na classificação de risco, que pode ser considerado como uma tecnologia leve-dura. Esse processo dinâmico de trabalho na emergência potencializa a imposição da interdisciplinaridade de saberes e a subjetividade de profissionais.

Um artigo aponta que o acolhimento com classificação de risco é como um dispositivo tecno-assistencial da enfermagem. Uma vez que, permite raciocinar e alterar os modos de operar a assistência; argumentar as relações de acesso à saúde, modelos de gestão e métodos de trabalho na emergência (Rates et al.2016).

Sobre o método de trabalho, o processo de enfermagem é um dos procedimentos científicos e sistematizados de assistência, alicerçado em teoria permite ao enfermeiro, dos serviços de emergência, realizar a consulta de enfermagem, de modo a investigar os problemas de saúde, diagnosticar, e identificar as respostas fisiológicas reais e de risco, planejar os resultados esperados, prescrever cuidados e avaliar sua assistência.

Aponta-se, ainda, Sobral et al. (2013), que o processo de enfermagem é a principal ferramenta de trabalho do enfermeiro, para planejar a assistência, dinamizar as ações de cuidado e sistematizar a consulta de forma a direcionar todos os esforços para a necessidade de saúde do cliente.

Mas no cotidiano de trabalho na emergência, a educação permanente pode ser facilmente introduzida, na promoção do aperfeiçoamento de habilidades técnicas e conhecimentos científicos. Ressalta-se assim, a importância dessa estratégia para viabilizar a consulta de enfermagem nesses espaços de cuidar.

\section{3 Tecnologia dura em enfermagem: sistema de informação e protocolos assistenciais de apoio ao cui- dado}

Constatou-se que na emergência, a consulta de enfermagem e protocolos assistências pode ser desenvolvido em software aplicativo, para apoiar o enfermeiro, a realizar tarefas especificas de cuidado. Que os sistemas de informação, são recursos providos pelas tecnologias computacionais, como suporte a automatização dos conhecimentos.

Para Soares et al. (2015), um sistema de informação de qualidade fornece informações essenciais para subsidiar pesquisas, implementar políticas e estratégias de cuidado; afim de prevenir doenças, promover a saúde e avaliar as ações.

Assim, os protocolos assistências e computacionais se combinam na consulta de enfermagem, são mecanismos tecnológicos de auxílio a sistematização do cuidado. Para atender a essa sistematização, os aplicativos produzidos especificamente para o processo de enfermagem na emergência pode favorecer a coletar e processar dados, transmitir informações clínicas e gerenciais; com o intuito de aprimorar o planejamento da assistência, a gerência do cuidado e a educação em enfermagem.

\section{CONSIDERAÇÕES FINAIS}

Foram analisados 30 artigos sobre os tipos de tecnologias de apoio ao ensino e práticas de enfermagem na emergência. De acordo com os dados apresentados neste estudo, foi possível perceber que são várias as tec- 
nologias utilizadas por esses profissionais, embora a leve e leve-dura no gerenciamento do cuidado são os mais presentes nos estudos.

Salienta-se que em alguns estudos identificaram-se as tecnologias cuidativas assistenciais, gerenciais e educacionais da enfermagem, de classificação leve e leve-dura, que permite uma maior aproximação entre o modelo de saúde biomédico e o modelo de cuidado centrado no cliente. Bem como a liderança situacional, o trabalho em equipe, a gestão de relacionamentos, a classificação de risco, o processo de enfermagem e a educação permanente.

Alguns autores consideram o atendimento do enfermeiro no acolhimento com classificação de risco à consulta de enfermagem. No entanto, sem indícios de qual teórico a intervenção de cuidado fundamenta-se.

Há a referência em estudos da presença do processo de enfermagem como método de cuidado, embasado em teorias de enfermagem, permite a prática cientifica de diagnosticar e prevenir agravos na emergência. Mas não se menciona de que forma esse modelo de cuidado pode ser desenvolvido no contexto da emergência hospitalar.

Refere-se a educação permanente como a principal estratégia educacional de empoderamento do enfermeiro na emergência, para aperfeiçoar técnicas e promover o conhecimento cientifico à equipe de enfermagem. Há citações de metodologias ativas como método relevante de ensino para problematizar questões vivenciadas no cotidiano profissional e possibilitar a construção coletiva de soluções inovadoras de cuidado.

Outro aspecto que merece notoriedade é o escasso número de inovações tecnológicas duras de apoio ao cuidado, elaboradas pela enfermagem e nenhuma alusão referente a patentes produzidas pela enfermagem na emergência. Diante disso, entende-se ser necessária a realização de estudos aprofundados acerca de estratégias de formação de recursos humanos em enfermagem que promova maior participação nesse segmentado empreendedor.

Com este estudo, sugere-se a inclusão de disciplina eletiva acerca da inovação e tecnologias em saúde na graduação em enfermagem; cursos de extensão sobre tecnologias e inovações no cuidado, no gerenciamento e no ensino de enfermagem; com vistas à capacitação e alcance da autonomia tecnológica por esses profissionais. Vale ressaltar a importância da implementação de núcleo de inovações tecnológicas na academia, com o intuito de potencializar projetos de saúde de impacto social, estimulando o pensamento inovador, e a articulação e o desenvolvimento de trabalhos científicos multidisciplinares entre acadêmicos e docentes, dos cursos de enfermagem, engenharia de produção e sistema de informação.

\section{REFERÊNCIAS}

AMTHUER, C; CUNHA, M. L. Manchester Triage System: main flowcharts, discriminators and outcomes of a pediatric emergency care. Revista Lat.-Am. Enfermagem. Aug. 29; 24: e2779. doi: 10.1590/15188345.1078.2779. Disponível em: //www.ncbi.nlm.nih.gov/pubmed/27579934. Acesso em 21 jul. 2017.

AZEVEDO, A. L. C. S; SCARPARO, A. F; CHAVES, L. D. P. Nurses care and management actions in emergency trauma cases. Invest. Educ. enfem. Mar 31(1):36-43, 2013. Disponível em: http://bases.bireme.br/cgibin/wxislind.exe/iah/online/?IsisScript=iah/iah. xis\&base=LILACS\&nextAction=lnk\&lang=p\&indexSearch=ID\&exprSearch=673519\&label=Nurses\%27\%20 care\%20and\%20management\%20actions\%20in\%20emergency\%20trauma\%20cases. Acesso em 20 jul. 2017

BARDIN L. Análise de conteúdo. Lisboa: Persona; 1977. 
BERTONCELLO, K. C. G; CAVALCANTI, C. A. K; ILHA, P. Análise do perfil do paciente como vítimas de múltiplos traumas. Cogitere Enferm. Out/Dez; 17(4): 717-23, 2012 Disponível em: <http://www.saude.ufpr.br/ portal/revistacogitare/wp-content/uploads/sites/28/2012/10/30380-111535-2-PB.pdf> . Acesso em: 20 jul. 2017

BERTOLO, V. F; RODRIGUES, C. D. S. Knowledge of cardiopulmonary resuscitation among pediatric emergency staff. Revista Enfermagem Uerj. V. 22, n. 4, 2014. Disponível em http://www.e-publicacoes.uerj. br/index.php/enfermagemuerj/article/view/15402/15742. Acesso em 20 jul. 2017.

BEZERRA, F. N; SILVA, T. M; RAMOS, V. P.Occupational stress of nurses in emergency care: an integrative review of the literature. ACTA Paul Enferm.;25(Special Issue 2):151-6. 2012. Disponível em: http://bases.bireme.br/cgi-bin/wxislind.exe/iah/online/?IsisScript=iah/iah. xis\&base=LILACS\&lang=p\&nextAction=lnk\&exprSearch=667524\&indexSearch=ID. Acesso em 20 jul. 2017

BORGES, L. R; PINHO, L. B. Atendimento à crise psíquica no pronto-socorro: visão de profissionais de enfermagem. Revista Gaúcha Enferm. Sept. Vol.33, n 3, 2012. Disponível em: http://www.scielo.br/scielo. php?script=sci_arttext\&pid=S1983-14472012000300004. Acesso em 21 jul. 2017.

CAMPOS C.J.G. Método de análise de conteúdos: ferramenta para a análise de dados qualitativos no campo da saúde. Rev. bras. enferm. [online]. 2004, vol.57, n.5, pp.611-614. ISSN 0034-7167. Disponível em: http://www. scielo.br/scielo.php?pid=S003471672004000500019\&script=sci_abstract\&tlng=pt. Acesso em 21 jul. 2017

CAVEIÃO, C; SANTOS, R. B; MONTEZELI, J. H. et al. Dor torácica: atuação do enfermeiro em um pronto atendimento de um hospital escola. R. Enferm. Cent. O. Min. Jan/abr; 4(1): 921-928, 2014. Disponível em: http://pesquisa.bvs.br/aps/resource/pt/bde-26605. Acesso em 20 jul. 2017

CORREA, M.B. et al. Tecnologia In: Cattani AD, organizador. Trabalho e Tecnologia: dicionário crítico. $2^{a}$ ed. Petrópolis (RJ): Vozes; 1999. P.251-57.

DOMINGOS, P. J; CHIANCA, T. C. M; SALAGADO, P. O. Predictive validity of theManchester Triage System: evaluation of outcomes of patients admitted to na emergency department. Revista Latino Americana Enfermagem. Nov.- Dec; 20(6):1041-1047, 2012. Disponível em: http://bases.bireme.br/cgibin/wxislind.exe/iah/online/?IsisScript=iah/iah. xis\&base=LILACS\&lang=p\&nextAction=lnk\&exprSearch=659786\&indexSearch=ID. Acesso em 20 jul. 2017

GEHLEN, G. C; LIMA, M. A. D. S. Nursing work in care practice at emergency care units in Porto Alegre/RS. Invest Educ Enferm.31(1): 26-35, 2013. Disponível em: http://www.lume.ufrgs.br/bitstream/ handle/10183/101833/000877394.pdf?sequence=1. Acesso em 20 jul. 2017.

JORGE,V. C; BARRETO, M. S; et al. Equipe de enfermagem e deteç̧ão de indicadores de agravamento em pacientes de pronto-socorro. Esc. Anna Nery. Oct./Dec; vol. 16 nº 4, 2012. Disponível em: http://www.scielo. br/scielo. php?script=sci_arttext\&pid=S1414-81452012000400018. Acesso em 21 jul. 2017.

JUNIOR, E. F. P; PAULA, V. G; SANTIAGO, L. C. et al. Implementação da classificação de risco em unidade de emergência de um hospital público do Rio de Janeiro - um relato de experiência. R. pesq.: cuid. Fundam. Online. Jan/Marc. 4(1): 2723-32, 2102. Disponível em: http://www.seer.unirio.br/index. php/cuidadofundamental/ article/view/1514/pdf_484. Acesso em 21 jul. 2017.

KOERICH, M.H.A. L; VIEIRA, R. H. G; SILVA, D. E. et al. Produção tecnológica brasileira na área de enfermagem: avanços e desafios. Rev. Gaúcha Enferm, 32(4): 736-43, 2011. Disponível em: http://www.scielo. br/scielo. php?script=sci_arttext\&pid=S198314472011000400014.acesso em 21 jul 2017 
LOUREIRO, L.H; ANDRIGHI, T. A. G; SEPP, V. J. et al. Estudo bibliométrico sobre produções cientificas na saúde com abordagem na inovação tecnológica: tendências e perspectivas. Rev. Acta Scientiae \& Technicae, vol.4, n², Dec. 2016 ISSN: 2317-8957

MAKITARIAN, F. F. P; ANGELO, M. Family's presence in the pediatric emergency room: opinion of health's professional. Revista Paul Pediatr. Oct/dec, 33(4): 460-466, 2015. Disponível em://www.ncbi.nlm.nih.gov/ pmc/articles/PMC4685567/. Acesso em 21 jul.2017

MARIA, M. A; QUADROS, F. A. A; GRASSI, M. F. O. Sistematização da assistência de enfermagem em serviços de urgência e emergência: viabilidade de implantação. Revista bras. enferm. Marc./Apr. Vol. 65, n ${ }^{\circ}$ 2, 2012. Disponível em: http://www.scielo.br/scielo. php?script=sci_arttext\&pid=S0034-71672012000200015. Acesso em 21 jul. 2017.

MENDES, I.A.C.; LEITE, J.L.; TREVIZAN, M.A. et al. A produção tecnológica e a interface com a enfermagem. Rev. Bras. Enf, v.55, n. 5, p.556-561, 2002. Disponível em:http://gruposdepesquisa.eerp.usp.br/gepecopen/ publicacoes/fd45c41b7471a47fdbae98d1f5c1a963.pdf. Acesso em 21 jul. 2017

MERHY, E.E. Em busca de ferramentas analisadoras das Tecnologias em Saúde: a informação e o dia a dia de um serviço, interrogando e gerindo trabalho em saúde. In: Merhy EE, Onoko, R, organizadores. Agir em Saúde: um desafio para o publico. 2ª ed. São Paulo (SP): Hucitec; 2002; 2002. P.113-150.

Ministério da Saúde. Disponível em: http://bvsms.saude.gov.br/bvs/saudelegis/gm/2012/prt1663_06_08_2012. html. Acesso em: 18 jun. 2017.

MORAIS, A. S; MELLEIRO, M. M; A qualidade da assistência de enfermagem em uma unidade de emergência: a percepção do usuário. Rev. Eletr. Enf. [Internet]. 2013 jan/mar;15(1):112-20. Disponível em: http://dx.doi. org/10.5216/ree.v15i1.15243. doi: 10.5216/ree.v15i1.15243.. Acesso em 21 jul. 2017.

OLIVEIRA, R. J. T; HERMIDA, P. M. V. et al. Care management in nursing within emergency care units. Invest. Educ Enferm.33(3):405-414, 2015. isponível em http://www.periodicos.capes.gov.br/?option=com_ pmetabusca $\& m n=88 \& s m n=88 \&$ type $=m \&$ metalib $=$ aHR0cDovL3JucC 1 wcmltby5ob3N0ZWQuZX

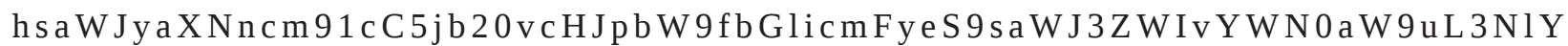
XJjaC5kbz8mdmlkPUNBUEVTX1YxJm1vZGU9QWR2YW5jZWQ=. Acesso em 21 jul. 2017.

OLIVEIRA, F. P; MAZZAIA, M. C; MARCOLAN, J. F. Symptoms of depression and intervening factors among nurses of emergency hospital services. Acta paul. Enferm. May/june, vol. 28, nº.3, 2015. Disponível em http://www.scielo.br/scielo. php?pid=S010321002015000300209\&script=sci_arttext\&tlng=en. Acesso em 21 jul. 2017

ROCHA, P. K; PRADO, M. L; WAL, M. L. et al. Cuidado e tecnologia: aproximações através do Modelo de Cuidado. Rev. Bras. Enf, v.61, n. 1, p.113-6, 2008. Disponível em: http://www.scielo.br/pdf/reben/v61n1/18. pdf. Acesso em 21 jul 2017

SANTOS, J. L. G; LIMA, M. A. D. S; KLOCK, P; ERDMANN, A. L. Concepções de enfermeiros sobre gerência do cuidado em um serviço de emergência: estudo exploratório-descritivo. Online Brazilian Journal of Nursing. May; 11(1), 2012. Disponivel em: http://www.objnursing.uff.br/index.php/nursing/article/view/3580/ html_2. Acesso em 20 jul. 2017.

SILVA, L. G; MATSUDA, L. M. Um olhar a qualidade no processo de atendimento em um serviço de urgência pública. Cienc Cuid Saude. 11(suplem.): 121-128, 2012.

Disponível em http://www.periodicos.uem.br/ojs/index. php/CiencCuidSaude/article/view/17063/pdf. Acesso em 21 jul. 2017. 
SOUZA, D. B; DALL' AGNOL, C. M. Public health emergency: social representations among managers of a university hospital. Revista Latino- Am. Enfermagem. July/Aug. Vol. 21, no 4, 2013. Disponível em: // www.ncbi.nlm.nih.gov/pubmed?term=Public\%20health\%20emergency\%3A\%20social\%20representations\%20 among\%20managers\%20of\%20a\%20university\%20hospital. Acesso em 21 jul. 2017.

NIETSCHE, E. A; DIAS, L. P. M; LEOPARDI, M.T.Tecnologias em Enfermagem: um saber prático? In: Anais do Seminário Nacional de Pesquisa em Enfermagem; 1999 maio; Gramado (RS), Brasil. Gramado (RS): ABEn-RS;1999.

NIETSCHE, E.A; TEIXEIRA, E.; MEDEIROS, H.P. Tecnologias cuidativo-educacionais: uma possibilidade para o empoderamento do(a) enfermeiro(a)?. 1.ed. Porto Alegre: Moriá, 2014.

POMPEO, D.A; ROSSI, L. A; GALVÃO C.M. Revisão integrativa: etapa inicial do processo de validação de diagnóstico de enfermagem. Acta paul. enferm. [online]. 2009, vol.22, n.4, pp.434-438. ISSN 1982-0194. Disponível em: http://www.scielo.br/scielo. php?pid=S010321002009000400014\&script=sci_abstract\&tlng=pt. Acesso em 21 jul. 2017

PRUDÊNCIO, C. P. G; MONTEIRO, R. A. N. et al. Percepção de enfermeira(o)s sobre acolhimento com classificação de risco no serviço de pronto atendimento. Revista Baiana de Enfermagem. Abr./jun, v.30, n. 2, p. 1-10, 2016. Disponível em: //portalseer.ufba.br/index.php/enfermagem/article/view/14917/pdf_46. Acesso em 21 jul. 2017.

RATES, H. F; ALVES, M; CAVALCANTE, R.B. O processo de trabalho do enfermeiro no acolhimento com classificação de risco. REME - Revista Min. Enferm. 20:e969, 2016. Disponível em http://www.reme.org.br/ artigo/detalhes/1105. Acesso em 21 jul. 2017

RESOLUÇÃOCOFENNo423/2012.Disponívelem:<http://www.cofen.gov.br/resoluo-cofen-n-4232012_8956. html>. Acesso em: 18 jun. 2017.

RODRIGUES, A.M.M. Por uma Filosofia da Tecnologia. In Grinspun MPSZ, organizador. Educação Tecnológica: desafios e perspectivas. $2^{\mathrm{a}}$ ed. São Paulo (SP): Cortez; 2001.

ROSSI, F.R. Tecnologias leves nos processos gerenciais do enfermeiro: contribuições para o cuidado humanizado. 2003. 118 f. Dissertação (metrado em enfermagem) - Universidade do rio Grande do Sul. Escola de Enfermagem. Porto Alegre. Disponível em: https://www.lume.ufrgs.br/bitstream/handle/10183/3845/000404791. pdf?sequence $=$. Acesso em 21 jul. 2017

SANTOS, F.P. A; NERY, A. A; MATUMOTO, S. A produção do cuidado a usuários com hipertensão arterial e as tecnologias em saúde. Rev.esc.enferm.USP, vol.47, nº1, 2013. Disponível em: http://dx.doi.org/10. 1590/ S0080-62342013000100014. Acesso em: 08/12/2016

SANTOS, M. T; LIMA, M. A. D. S; ZUCATTI, P. B. Serviços de emergência amigos do idoso no Brasil: condições necessárias para o cuidado. Revista Esc. Enferm USP. 50(4): 592-599, 2016. Disponível em: https:// www.lume.ufrgs.br/bitstream/handle/10183/149447/001003791.pdf?sequence=1 . Acesso em 21 jul. 2017

SANTOS, J. L. G; LIMA, M. A. D. S; PESTANA, A. L. et al. Strategies used by nurses to promote teamwork in na emergency room. Revista Gaúcha de Enfermagem. Feb 26. Vol.37, nº 1, 2016. Disponível em http://www. scielo.br/scielo. php?pid=S1983-14472016000100402\&script=sci_arttext\&tlng=en. Acesso em 21 jul. 2017

SANTOS, J. L. G. et al. Estratégias utilizadas pelos enfermeiros para promover o trabalho em equipe em um serviço de emergência. Revista Gaúcha de Enfermagem, mar; 37(1), 2016.Disponível em: http://www.scielo. br/scielo.php?pid=S1983-14472016000100402\&script=sci_abstract\&tlng=pt. Acesso em 21 jul. 2017 
SEIGNEMARTIN, B. A; JESUS, L. R. et al. Avaliação da qualidade das anotações de enfermagem no pronto atendimento de um hospital escola. Revista Rene. 14(6):1123-32, 2013. Disponível em: http://periodicos.ufc.br/ rene/article/view/3724/2944. Acesso em 21 jul. 2017

SILVA, D.C; ALVIM, N. A. T; FIGUEIREDO, P. A. Tecnologias leves em saúde e sua relação com o cuidado de enfermagem hospitalar. Escola Anna Nery - Revista Enfermagem, 12(2): 291-8, 2008. Disponível em: http:// www.scielo.br/pdf/ean/v12n2/v12n2a14. Acesso em 21 jul. 2017

SILVA, M. F. N; OLIVEIRA, G. N. et al. Assessment and risk classification protocol for patients in emergency units. Revista Latino-Am. Enfermagem. Mar/abr; vol. 22, nº 2, 2014. Disponível em: //www.ncbi.nlm.nih.gov/ pubmed/26107828. Acesso em 21 jul. 2017

SILVA, P. L; PAIVA, L; FARIA, V. B. et al. Triage in na adult emergency service: patient satisfaction. Revista Esc. Enferm. USP. May/june, vol. 50, no. 3, 2016. Disponível em http://www.scielo.br/scielo. php?script=sci_ arttext\&pid=S0080-62342016000300427. Acesso em 21 jul. 2017

SOARES, L. S; SOUZA, D. A. C. M. et al. Caracterização das vítimas de traumas por acidente com motocicleta internadas em um hospital público. Revista enfermagem - UERJ, jan/fev; 23(1):115-21, 2015. Disponível em: http://www.facenf.uerj.br/v23n1/v23n1a19.pdf. Acesso em 20 jul. 2017.

SOBRAL, P. H. A. F. et al. Atuação de enfermagem em serviços de emergência: revisão integrativa. Journal of Research Fundamental Care On Line, out/dez; 5(4):396-07, 2013. Disponível em: http://www.seer.unirio.br/ index.php/cuidadofundamental/article/view/1655/pdf_893. Acesso em 20 jul. 2017.

SOUZA, M. L; SARTOR, V. V. B; PADILHA, M. I. C. S.et al. O Cuidado em Enfermagem: uma Aproximação Teórica. Texto Contexto Enferm, 14(2):266-70, 2005. Disponível em: http://www.scielo.br/pdf/tce/v14n2/ a15v14n2. Acesso em 21 jul. 2017

VIANNA, I. O. Metodologia do trabalho científico: um enfoque didático da produção científica. São Paulo: EPU, 2001.

VIEIRA, A. C; BERTONCELLO, K. C. G; GIRONDI, J. B. R. Perception of emergency nurses in using a chest pain assessment protocol. Texto Contexto Enferm. 25(1):e1830014, 2016. Disponível em: http://www.scielo. br/pdf/tce/v25n1/0104-0707-tce-25-01-1830014.pdf. Acesso em 21 jul. 2017

WHITTEMORE R; KNAFL K. The Integrative Review:up-dated Methodology. J Adv Nurs. 52(5): 546-53, 2005. Disponível em: http://citeseerx.ist.psu.edu/viewdoc/download?doi=10.1.1.465.9393\&rep=rep1\&type=pdf. Acesso em 21 jul. 2017

ZANDOMENIGHI, R. C; MOURO, D. L. et al. Cuidados intensivos em um serviço hospitalar de emergência: desafios para os enfermeiros. REME - Revista Min. Enferm. Abr/jun; 18(2): 404414, 2014. Disponível em http://bases.bireme.br/cgi-bin/wxislind.exe/iah/online/?IsisScript=iah/iah. xis\&src=google\&base=BDENF\&lang=p\&nextAction=lnk\&exprSearch=25729\&indexSearch=ID. Acesso em 21 jul. 2017. 